\title{
Analytic continuation of multiple zeta-functions and their values at non-positive integers
}

\author{
by \\ Shigeki Akiyama (Niigata), Shigeki Egami (Toyama) \\ and Yoshio Tanigawa (Nagoya)
}

1. Introduction. The multiple zeta values due to D. Zagier are defined by

$$
\zeta_{k}\left(s_{1}, \ldots, s_{k}\right)=\sum_{0<n_{1}<\ldots<n_{k}} \frac{1}{n_{1}^{s_{1}} \ldots n_{k}^{s_{k}}}
$$

for positive integers $s_{i}(i=1, \ldots, k)$ and $s_{k} \geq 2$. These values have a certain connection with topology and physics, and algebraic relations among them are extensively studied (see [18], [19], [6], [7] and [14]). Recently, Y. Ohno developed a unified algebraic relation in [16]. It is also interesting to consider it for complex variables $s_{i}$.

In this paper, we deal with analytic continuation of $\zeta_{k}\left(s_{1}, \ldots, s_{k}\right)$. Analytic continuation of $\zeta_{2}\left(s_{1}, s_{2}\right)$ was proved by F. V. Atkinson [5] with applications to the study of the asymptotic behavior of the "mean values" of zeta-functions. See also Y. Motohashi [15] and M. Katsurada \& K. Matsumoto [13]. In [4], T. Arakawa \& M. Kaneko used analytic continuation of $\zeta_{k}\left(s_{1}, \ldots, s_{k}\right)$ as a function of one variable $s_{k}$ when $s_{1}, \ldots, s_{k-1}$ are positive integers, and discussed the relation among generalized Bernoulli numbers. On the other hand, S. Egami discussed the relationship among various multiple zeta-functions introduced by E. W. Barnes, T. Shintani and D. Zagier. (See [9] and [10].)

However, for a general $k$, we have not found the proof of analytic continuation of $\zeta_{k}\left(s_{1}, \ldots, s_{k}\right)$ as a function of $k$ variables in the literature (but see the comment of Zagier [18, p. 509, lines 14-19]). We shall show that the multiple zeta-function can be continued analytically to $\mathbb{C}^{k}$ and discuss interesting properties of multiple zeta values at non-positive integers.

2000 Mathematics Subject Classification: Primary 11M41; Secondary 32Dxx. 
The authors wish to express their gratitude to the referee for valuable comments on the earlier version of the present paper. The third author also thanks Professor Aleksandar Ivić for useful comments.

REMARK 1. After submitting the first version of our paper, we found a recent work of J. Q. Zhao [20] treating analytic continuation of multiple zeta-functions. This fact was also pointed out by the referee. With the help of the theory of generalized functions in the sense of I. M. Gel'fand and G. E. Shilov, J. Q. Zhao gave the possible singularities as well as the residues. However our method is apparently simpler and reveals the exact location of singularities, which seems to be an advantage.

2. Analytic continuation. Let $l$ and $m$ be positive integers. Define an entire function by

$$
\phi_{l}(m, s)=\sum_{n=1}^{m} \frac{1}{n^{s}}-\left\{\frac{m^{1-s}-1}{1-s}+\frac{1}{2 m^{s}}-\sum_{q=1}^{l} \frac{(s)_{q} a_{q}}{m^{s+q}}+\zeta(s)-\frac{1}{s-1}\right\}
$$

with $(s)_{n}=s(s+1) \ldots(s+n-1)$ and $a_{q}=B_{q+1} /(q+1)$ !. Here $B_{q}$ are Bernoulli numbers defined by $z /\left(e^{z}-1\right)=\sum_{q=0}^{\infty} B_{q} z^{q} / q$ ! and $\zeta(s)$ is the Riemann zeta-function. By using the Euler-Maclaurin summation formula, we have $\phi_{l}(m, s)=O\left(\left|(s)_{l+1}\right| m^{-\Re(s)-l-1}\right)$ when $s$ is a complex number. Considering $s$ as a complex variable and $m \rightarrow \infty$, we get an analytic continuation of $\zeta(s)$ in $\Re(s+l+1)>0$. Note that (1) is also valid as $s \rightarrow 1$, if we replace $\left(m^{1-s}-1\right) /(1-s)$ by its limit $\log m$.

This is one of the oldest ways of the analytic continuation of the Riemann zeta-function, which provides us with a method of numerical calculations in the critical strip $0<\Re(s)<1$ (cf. [8], [12]). It does not give us the celebrated functional equation of $\zeta(s)$ directly, but it is possible to derive it by more precise observations (see Chapter 2 of [17]). Hereafter we will use (1) in the form

$$
\sum_{n=m+1}^{\infty} \frac{1}{n^{s}}=-\phi_{l}(m, s)+\frac{m^{1-s}}{s-1}-\frac{1}{2 m^{s}}+\sum_{q=1}^{l} \frac{(s)_{q} a_{q}}{m^{s+q}}
$$

for $\Re(s)>1$. Consider the multiple zeta-function in two variables

for $\Re\left(s_{i}\right)>1(i=1,2)$. By $(2)$,

$$
\zeta_{2}\left(s_{1}, s_{2}\right)=\sum_{0<n_{1}<n_{2}} \frac{1}{n_{1}^{s_{1}} n_{2}^{s_{2}}}
$$

$$
\begin{aligned}
\zeta_{2}\left(s_{1}, s_{2}\right) & =\sum_{n_{1}=1}^{\infty} \frac{1}{n_{1}^{s_{1}}} \sum_{n_{2}=n_{1}+1}^{\infty} \frac{1}{n_{2}^{s_{2}}} \\
& =\sum_{n_{1}=1}^{\infty} \frac{1}{n_{1}^{s_{1}}}\left\{-\phi_{l}\left(n_{1}, s_{2}\right)+\frac{n_{1}^{1-s_{2}}}{s_{2}-1}-\frac{1}{2 n_{1}^{s_{2}}}+\sum_{q=1}^{l} \frac{\left(s_{2}\right)_{q} a_{q}}{n_{1}^{s_{2}+q}}\right\}
\end{aligned}
$$




$$
\begin{aligned}
= & \frac{\zeta\left(s_{1}+s_{2}-1\right)}{s_{2}-1}-\frac{\zeta\left(s_{1}+s_{2}\right)}{2} \\
& +\sum_{q=1}^{l}\left(s_{2}\right)_{q} a_{q} \zeta\left(s_{1}+s_{2}+q\right)-\sum_{n_{1}=1}^{\infty} \frac{\phi_{l}\left(n_{1}, s_{2}\right)}{n_{1}^{s_{1}}}
\end{aligned}
$$

for $\Re\left(s_{i}\right)>1(i=1,2)$. The terms on the right hand side except the last one have meromorphic continuations. The last sum is absolutely convergent, and hence holomorphic, in $\Re\left(s_{1}+s_{2}+l\right)>0$. Thus we now have a meromorphic continuation of $\zeta_{2}\left(s_{1}, s_{2}\right)$ to $\Re\left(s_{1}+s_{2}+l\right)>0$. Since we can choose $l$ arbitrarily large, we get a meromorphic continuation of $\zeta_{2}\left(s_{1}, s_{2}\right)$ to $\mathbb{C}^{2}$, which is holomorphic in

$$
\left\{\left(s_{1}, s_{2}\right) \in \mathbb{C}^{2} \mid s_{2} \neq 1, s_{1}+s_{2} \notin\{2,1,0,-2,-4,-6, \ldots\}\right\} .
$$

One can easily see that this trick can be applied to a multiple zetafunction with $k$ variables. In fact,

$$
\begin{aligned}
\zeta_{k}( & \left.s_{1}, \ldots, s_{k}\right) \\
= & \sum_{n_{1}=1}^{\infty} \frac{1}{n_{1}^{s_{1}}} \sum_{n_{2}=n_{1}+1}^{\infty} \frac{1}{n_{2}^{s_{2}}} \ldots \sum_{n_{k-1}=n_{k-2}+1}^{\infty} \frac{1}{n_{k-1}^{s_{k-1}}} \sum_{n_{k}=n_{k-1}+1}^{\infty} \frac{1}{n_{k}^{s_{k}}} \\
= & \sum_{n_{1}=1}^{\infty} \frac{1}{n_{1}^{s_{1}}} \sum_{n_{2}=n_{1}+1}^{\infty} \frac{1}{n_{2}^{s_{2}}} \ldots \sum_{n_{k-1}=n_{k-2}+1}^{\infty} \frac{1}{n_{k-1}^{s_{k-1}}} \\
& \times\left\{-\phi_{l}\left(n_{k-1}, s_{k}\right)+\frac{n_{k-1}^{1-s_{k}}}{s_{k}-1}-\frac{1}{2 n_{k-1}^{s_{k}}}+\sum_{q=1}^{l} \frac{\left(s_{k}\right)_{q} a_{q}}{n_{k-1}^{s_{k}+q}}\right\} \\
= & \frac{\zeta_{k-1}\left(s_{1}, \ldots, s_{k-2}, s_{k-1}+s_{k}-1\right)}{s_{k}-1}-\frac{\zeta_{k-1}\left(s_{1}, \ldots, s_{k-2}, s_{k-1}+s_{k}\right)}{2} \\
& +\sum_{q=1}^{l}\left(s_{k}\right)_{q} a_{q} \zeta_{k-1}\left(s_{1}, \ldots, s_{k-2}, s_{k-1}+s_{k}+q\right) \\
& -\sum_{0<n_{1}<\ldots<n_{k-1}} \frac{\phi_{l}\left(n_{k-1}, s_{k}\right)}{n_{1}^{s_{1}} \ldots n_{k-1}^{s_{k-1}}}
\end{aligned}
$$

for $\Re\left(s_{i}\right)>1(i=1, \ldots, k)$. Since

$$
\sum_{0<n_{1}<\ldots<n_{k-1}} \frac{\phi_{l}\left(n_{k-1}, s_{k}\right)}{n_{1}^{s_{1}} \ldots n_{k-1}^{s_{k-1}}} \ll \sum_{n_{k-1}} \frac{n_{k-1}^{-l-\Re\left(s_{k}\right)+k-3}}{n_{k-1}^{L}}
$$

with

$$
L=\Re\left(s_{k-1}\right)+\sum_{\substack{1 \leq j \leq k-2 \\ \Re\left(s_{i}\right) \leq 0}} \Re\left(s_{i}\right),
$$


the last summation is absolutely convergent in the set

$$
l-k+2+\Re\left(s_{k}\right)+\Re\left(s_{k-1}\right)+\sum_{\substack{1 \leq i \leq k-2 \\ \Re\left(s_{i}\right) \leq 0}} \Re\left(s_{i}\right)>0 .
$$

Since $l$ can be taken arbitrarily large, we get an analytic continuation of $\zeta_{k}\left(s_{1}, \ldots, s_{k}\right)$ to $\mathbb{C}^{k}$. Now we consider the set of singularities. For simplicity, we put $(s)_{0}=1$. Then the "singular part" of $\zeta_{2}\left(s_{1}, s_{2}\right)$ is given by

$$
\frac{\zeta\left(s_{1}+s_{2}-1\right)}{s_{2}-1}+\sum_{q_{1} \geq 0} \frac{a_{q_{1}}\left(s_{2}\right)_{q_{1}}}{s_{1}+s_{2}+q_{1}-1} .
$$

Note that this sum is formal and only indicates local singularities. From this expression, we see that

$$
s_{2}=1, \quad s_{1}+s_{2} \in\{2,1,0,-2,-4,-6, \ldots\}
$$

forms the set of all singularities. For the case $\zeta_{3}\left(s_{1}, s_{2}, s_{3}\right)$, by using the singular part of $\zeta_{2}$, we see that singularities lie on

$$
s_{3}=1, \quad s_{2}+s_{3} \in\{2,1,0,-2,-4,-6, \ldots\}
$$

and

$$
s_{1}+s_{2}+s_{3} \in\{3,2,1,0,-1,-2,-3, \ldots\} .
$$

We want to show that these are all singularities. It suffices to show that no singularities defined by one of the above equations will identically vanish. This can be shown by changing variables:

$$
u_{1}=s_{1}, \quad u_{2}=s_{2}+s_{3}, \quad u_{3}=s_{3} .
$$

In fact, we see that the singular part of $\zeta_{3}\left(u_{1}, u_{2}-u_{3}, u_{3}\right)$ is given by

$$
\frac{1}{u_{3}-1} \zeta_{2}\left(u_{1}, u_{2}-1\right)+\sum_{q_{2} \geq 0}\left(u_{3}\right)_{q_{2}} a_{q_{2}} \zeta_{2}\left(u_{1}, u_{2}+q_{2}\right) .
$$

By this expression we see that the singularities of $\zeta_{2}\left(u_{1}, u_{2}+q\right)$ are summed with functions of $u_{3}$ of different degree. Thus these singularities, as a weighted sum by another variable $u_{3}$, will not vanish identically. Similarly, we see

THEOREM 1. The multiple zeta-function $\zeta_{k}\left(s_{1}, \ldots, s_{k}\right)$ continues meromorphically to $\mathbb{C}^{k}$ and has singularities on

$$
s_{k}=1, \quad s_{k-1}+s_{k}=2,1,0,-2,-4, \ldots
$$

and

$$
\sum_{i=1}^{j} s_{k-i+1} \in \mathbb{Z}_{\leq j} \quad(j=3,4, \ldots, k),
$$

where $\mathbb{Z}_{\leq j}$ is the set of integers less than or equal to $j$. 
3. Zeta values at non-positive integers. In this section, we use the notation $(s)_{0}=1$ and $(s)_{-1}=1 /(s-1)$ for the sake of simplicity. We also put $a_{q}=B_{q+1} /(q+1)$ ! for $q=0$ and -1 as in Section 2 . A point of $\mathbb{C}^{n}(n \geq 2)$ is said to be a point of indeterminacy of a meromorphic function if both the local denominator and the local numerator vanish there. See p.164 of [1] for the precise definition. For instance, let $f\left(s_{1}, s_{2}\right)=s_{1} /\left(s_{1}+s_{2}\right)$. Then $s_{1}=s_{2}=0$ is a point of indeterminacy of $f$. So the value of $f$ at $(0,0)$ depends on a limiting process, for example $\lim _{s_{2} \rightarrow 0} \lim _{s_{1} \rightarrow 0} f\left(s_{1}, s_{2}\right)=0$ while $\lim _{s_{1} \rightarrow 0} \lim _{s_{2} \rightarrow 0} f\left(s_{1}, s_{2}\right)=1$.

Let $r_{i}(i=1, \ldots, k)$ be non-negative integers. Recall from Theorem 1 that each point $\left(-r_{1},-r_{2}, \ldots,-r_{k}\right)$ except when $k=2$ and $r_{1}+r_{2}$ is odd, lies on the set of singularities. Moreover, such a point is a point of indeterminacy. To prove this, it suffices to show that $\zeta_{k}$ has a finite value at $\left(-r_{1},-r_{2}, \ldots,-r_{k}\right)$ by a specific limiting process. Now we give the definition which we will employ in this paper.

Definition. We define the multiple zeta values at non-positive integers by

$$
\zeta_{k}\left(-r_{1}, \ldots,-r_{k}\right)=\lim _{s_{1} \rightarrow-r_{1}} \ldots \lim _{s_{k} \rightarrow-r_{k}} \zeta_{k}\left(s_{1}, \ldots, s_{k}\right) .
$$

From (4) and the above definition, we have

$$
\begin{aligned}
\zeta_{k}\left(-r_{1}, \ldots,\right. & \left.-r_{k}\right) \\
& =\sum_{q=-1}^{r_{k}}\left(-r_{k}\right)_{q} a_{q} \zeta_{k-1}\left(-r_{1}, \ldots,-r_{k-2},-r_{k-1}-r_{k}+q\right) .
\end{aligned}
$$

Here we used the fact that $\phi_{r}(m, l)=0$ for $l \geq r$. This formula shows that the value $\zeta_{k}\left(-r_{1}, \ldots,-r_{k}\right)$ is determined recursively as a finite number, hence each point $\left(-r_{1}, \ldots,-r_{k}\right)$ is a point of indeterminacy. The formula (6) also gives us a simple way of calculating the multiple zeta values $\zeta_{k}\left(-r_{1}, \ldots,-r_{k}\right)$. For example, we have $\zeta_{2}(0,0)=1 / 3, \zeta_{3}(0,0,0)=-1 / 4$, $\zeta_{4}(0,0,0,0)=1 / 5, \zeta_{2}(-1,-1)=1 / 360, \zeta_{3}(-1,-1,-1)=83 / 30240$. One may expect that $\zeta_{k}(0, \ldots, 0)=(-1)^{k} /(1+k)$. This will be proved in [2]. Here we show some other interesting properties.

THEOREM 2. Let $r_{i}(i=1, \ldots, k)$ be non-negative integers. Then the value $\zeta_{k}\left(-r_{1}, \ldots,-r_{k}\right)$ is a rational number whose denominator has prime factors less than or equal to $1+k+\sum_{i=1}^{k} r_{i}$.

Proof. It is well known that $\zeta(0)=-1 / 2, \zeta(-2 r)=0$ and $\zeta(1-2 r)=$ $-B_{2 r} /(2 r)$ for positive integers $r$. By the theorem of von Staudt \& Clausen, the assertion for $k=1$ is obvious. From (6), the proof is completed by induction on $k$. 
Theorem 3. Let $r_{i}(i=1, \ldots, k)$ be positive integers and $n_{i}(i=1, \ldots, k)$ be non-negative integers. If $\sum_{i=1}^{k}\left(r_{i}+n_{i}+1\right)$ is odd then

$$
\sum_{\sigma \in \mathfrak{S}_{k}} \operatorname{sgn}(\sigma) \zeta_{k}\left(-r_{\sigma(1)}-n_{1}, \ldots,-r_{\sigma(k)}-n_{k}\right)=0,
$$

where $\mathfrak{S}_{k}$ is the symmetric group of degree $k$ and $\operatorname{sgn}(\sigma)$ is the signature of $\sigma \in \mathfrak{S}_{k}$.

The statement is trivial when $r_{i}$ are not distinct. We give some examples when $n_{1}=n_{2}=n_{3}=0$ before proving the theorem:

$$
\begin{aligned}
& \zeta_{2}(-1,-2)-\zeta_{2}(-2,-1)=-\frac{1}{240}+\frac{1}{240}=0, \\
& \zeta_{3}(-1,-2,-3)+\zeta_{3}(-2,-3,-1)+\zeta_{3}(-3,-1,-2) \\
& \quad-\zeta_{3}(-1,-3,-2)-\zeta_{3}(-2,-1,-3)-\zeta_{3}(-3,-2,-1) \\
& =-\frac{101}{100800}+\frac{149}{302400}+\frac{107}{302400}+\frac{19}{30240}+\frac{17}{43200}-\frac{131}{151200}=0 .
\end{aligned}
$$

Proof of Theorem 3. We only prove the case $n_{1}=\ldots=n_{k}=0$. The generalization is quite easy and is left to the reader. Let

$$
I_{k}=\left\{\left(-r_{1}, \ldots,-r_{k}\right) \mid r_{i} \text { are positive integers and } \sum_{i=1}^{k}\left(r_{i}+1\right) \text { is odd }\right\} .
$$

For $1 \leq a<b \leq k$, we define a vector space $\mathfrak{F}_{k}(a, b)$ to consist of the $\mathbb{C}$-valued functions $f\left(\xi_{1}, \ldots, \xi_{k}\right)$ such that

$$
\sum_{\sigma \in \mathfrak{S}_{a: b}} \operatorname{sgn}(\sigma) f\left(-r_{\sigma(1)}, \ldots,-r_{\sigma(k)}\right)=0
$$

for any $\left(-r_{1}, \ldots,-r_{k}\right) \in I_{k}$, where $\mathfrak{S}_{a: b}$ is the subgroup of $\mathfrak{S}_{k}$ of elements which stabilize $\{1, \ldots, k\} \backslash\{a, a+1, \ldots, b\}$. Our task is to show that the function $\zeta_{k}\left(\xi_{1}, \ldots, \xi_{k}\right)$ is in $\mathfrak{F}_{k}(1, k)$. Considering the coset decomposition $\mathfrak{S}_{k} / \mathfrak{S}_{a: b}$, we see that $\mathfrak{F}_{k}(a, b)$ is a subspace of $\mathfrak{F}_{k}(1, k)$. Thus it is enough to show that the multiple zeta-function lies in a sum of subspaces $\mathfrak{F}_{k}(a, b)$.

First we prove the case $k \leq 3$. The assertion (7) is valid when $k=1$, since $\zeta(-2 r)=0$ for any positive integers $r$. When $k=2$ and $\left(-r_{1},-r_{2}\right) \in I_{2}$, we have

$$
\zeta_{2}\left(-r_{1},-r_{2}\right)=\sum_{q=-1}^{r_{2}}\left(-r_{2}\right)_{q} a_{q} \zeta\left(-r_{1}-r_{2}+q\right)=-\frac{1}{2} \zeta\left(-r_{1}-r_{2}\right),
$$

which shows the assertion for $k=2$. When $k=3$ and $\left(-r_{1},-r_{2},-r_{3}\right) \in I_{3}$, we see from (8) that

$$
\zeta_{3}\left(-r_{1},-r_{2},-r_{3}\right)=\sum_{q=-1}^{r_{3}}\left(-r_{3}\right)_{q} a_{q} \zeta_{2}\left(-r_{1},-r_{2}-r_{3}+q\right)
$$




$$
=-\frac{1}{2} \zeta_{2}\left(-r_{1},-r_{2}-r_{3}\right)-\frac{1}{2} \sum_{\substack{q=-1 \\ q \text { odd }}}^{r_{3}}\left(-r_{3}\right)_{q} a_{q} \zeta\left(-r_{1}-r_{2}-r_{3}+q\right) .
$$

Hence $\zeta_{3}\left(\xi_{1}, \xi_{2}, \xi_{3}\right) \in \mathfrak{F}_{3}(2,3)+\mathfrak{F}_{3}(1,2)$.

Let $k \geq 3$ and $\left(-r_{1}, \ldots,-r_{k}\right) \in I_{k}$. Then by induction on $k$, we can easily see that

$$
\begin{aligned}
\zeta_{k}\left(\xi_{1}, \ldots, \xi_{k}\right)+\frac{1}{2} \zeta_{k-1}\left(\xi_{1}, \ldots, \xi_{k-2}, \xi_{k-1}+\xi_{k}\right) \\
\quad \in \mathfrak{F}_{k}(1,2)+\mathfrak{F}_{k}(2,3)+\ldots+\mathfrak{F}_{k}(k-2, k-1) .
\end{aligned}
$$

The assertion of the theorem follows immediately from (10).

Suppose that $k=3, r_{i}$ are non-negative integers, $r_{1}>0$ and $r_{1}+r_{2}+r_{3}$ is even. Then from (8) and (9), we have

$$
\zeta_{3}\left(-r_{1},-r_{2},-r_{3}\right)=-\frac{1}{2}\left\{\zeta_{2}\left(-r_{1}-r_{2},-r_{3}\right)+\zeta_{2}\left(-r_{1},-r_{2}-r_{3}\right)\right\} .
$$

One may expect that symmetric expressions like (8) and (11) would give us a deeper understanding of Theorem 3. Further calculation suggests the conjecture given below. To state it, we prepare some notation. Let $S$ be the ordered index set $\{1, \ldots, k\}$ of $k$ elements and let $\mathcal{D}_{l}^{k}$ be the set of all ways of dividing $S$ into $l$ parts. Clearly $\mathcal{D}_{l}^{k}$ has $\left(\begin{array}{c}k-1 \\ l-1\end{array}\right)$ elements. An element $J$ in $\mathcal{D}_{l}^{k}$ can be expressed as

$$
J=\left(1, \ldots, i_{1}\left|i_{1}+1, \ldots, i_{2}\right| i_{2}+1, \ldots, i_{l-1} \mid i_{l-1}+1, \ldots, k\right) .
$$

Let $A=\left(-r_{1}, \ldots,-r_{k}\right)$ be a sequence of $k$ non-positive integers. For $J \in \mathcal{D}_{l}^{k}$ as above, we set

$$
A^{J}=\left(-r_{1}-r_{2}-\ldots-r_{i_{1}},-r_{i_{1}+1}-\ldots-r_{i_{2}}, \ldots,-r_{i_{l-1}+1}-\ldots-r_{k}\right)
$$

and

$\zeta_{l}\left(A^{J}\right)=\zeta_{l}\left(-r_{1}-r_{2}-\ldots-r_{i_{1}},-r_{i_{1}+1}-\ldots-r_{i_{2}}, \ldots,-r_{i_{l-1}+1}-\ldots-r_{k}\right)$.

Now we can state

CONJeCture $\left({ }^{1}\right)$. Let $r_{i}$ be non-negative integers such that $r_{1}>0$ and $\sum_{i=1}^{k}\left(r_{i}+1\right)$ is odd. Let $A=\left(-r_{1}, \ldots,-r_{k}\right)$. Then

$$
\zeta_{k}(A)=-2 \sum_{j=1}^{k-1}\left(2^{j+1}-1\right) \frac{B_{j+1}}{j+1}\left(\sum_{J \in \mathcal{D}_{k-j}^{k}} \zeta_{k-j}\left(A^{J}\right)\right) .
$$

The coefficients in the first summation of (12) were inferred from the home page 'Sloane's On-Line Encyclopedia of Integer Sequences'.

$\left({ }^{1}\right)$ Addendum for the revised version: We have succeeded in proving the validity of this Conjecture. See [2] for details. 
TheOREM 4. For a positive integer $r$, we have

$$
\frac{\zeta(-4 r-1)}{\zeta_{2}(-2 r,-2 r)}=(2 r+1)\left(\begin{array}{c}
4 r+2 \\
2 r+1
\end{array}\right) \text {. }
$$

Proof. From (6) and the definition of $a_{q}$, we have

$$
\zeta_{2}(-2 r,-2 r)=\frac{B_{4 r+2}}{2(2 r+1)^{2}}+\frac{1}{2 r+1} \sum_{j=1}^{r}\left(\begin{array}{c}
2 r+1 \\
2 j
\end{array}\right) \frac{B_{2 j} B_{4 r+2-2 j}}{4 r+2-2 j} .
$$

We note the following identity for Bernoulli numbers:

$$
2(2 r+1) \sum_{j=0}^{r}\left(\begin{array}{c}
2 r+1 \\
2 j
\end{array}\right) \frac{B_{2 j} B_{4 r+2-2 j}}{4 r+2-2 j}+\frac{((2 r+1) !)^{2}}{(4 r+2) !} B_{4 r+2}=0,
$$

obtained by putting $m=n=2 r+1$ and $x=0$ in [3, p. 276, 19(b)]. Hence, we have

$$
\zeta_{2}(-2 r,-2 r)=-\frac{1}{2(2 r+1)^{2}} \cdot \frac{((2 r+1) !)^{2}}{(4 r+2) !} B_{4 r+2} .
$$

On the other hand, $\zeta(-4 r-1)=-B_{4 r+2} /(4 r+2)$, and this gives the assertion of Theorem 4 .

Finally we want to add several remarks.

REMARK 2. There are many other possibilities for the definition of multiple zeta values at non-positive integers. For instance, define

$$
\zeta_{k}^{*}\left(-r_{1}, \ldots,-r_{k}\right)=\lim _{\varepsilon \rightarrow 0} \zeta_{k}\left(-r_{1}+\varepsilon, \ldots,-r_{k}+\varepsilon\right) .
$$

When $k=2$, this is equivalent to defining

$$
\zeta_{2}^{*}\left(-r_{1},-r_{2}\right)=\sum_{q=-1}^{r_{1}}\left(-r_{2}\right)_{q} a_{q} \zeta\left(-r_{1}-r_{2}+q\right)+\frac{(-1)^{r_{1}} r_{1} ! r_{2} ! a_{r_{1}+r_{2}+1}}{2}
$$

for non-negative integers $r_{i}(i=1,2)$. This definition seems to be better than our former definition at least when $k=2,3$. In fact, when $r_{1}+r_{2}$ is odd we have $\zeta_{2}\left(-r_{1},-r_{2}\right)=\zeta_{2}^{*}\left(-r_{1},-r_{2}\right)$ and

$$
\zeta_{2}^{*}\left(-2 u_{1},-2 u_{2}\right)+\zeta_{2}^{*}\left(-2 u_{2},-2 u_{1}\right)=0
$$

for positive integers $u_{i}(i=1,2)$. In particular, we have $\zeta_{2}^{*}(-2 u,-2 u)=0$ with a positive integer $u$. We can also find a recursive formula for $k=3$ and show that

$$
\zeta_{3}^{*}(-2 u,-2 v,-2 w)+\zeta_{3}^{*}(-2 w,-2 v,-2 u)=0
$$

for positive integers $u, v, w$. However, in the general case, it seems difficult to construct a recursive formula like (13), since there are a lot of singularities to be taken into account. One may hope that 


$$
\zeta_{k}^{*}(-2 u,-2 u, \ldots,-2 u)=0
$$

for a positive integer $u$.

REMARK 3. The set of points of indeterminacy forms a $(k-2)$-dimensional holomorphic subvariety of $\mathbb{C}^{k}$, by p. 166 of [11]. We have shown that all non-positive points $\left(-r_{1}, \ldots,-r_{k}\right)$ are actually on this subvariety, but there are another type of integer points in this set. For instance, it will be shown in [2] that $\left(-r_{1}, \ldots,-r_{k-1}, 1\right)$ is a point of indeterminacy whose multiple zeta value in our sense is rational, when $r_{i} \in \mathbb{Z}_{\geq 0}$ and not all $r_{i}$ is zero. Also we have

$$
\zeta_{3}(4,-3,-2)=-\frac{461}{2520}-\frac{\pi^{2}}{144}+\frac{\pi^{4}}{45360}+\frac{\zeta(3)}{420} .
$$

We have not been able yet to determine the whole set of such integer points.

REMARK 4. We can easily apply the Euler-Maclaurin summation formula to more general zeta-functions. For instance, let $\alpha_{i}>-1(i=1, \ldots, k)$ be real numbers and $\chi_{i}(i=1, \ldots, k)$ the Dirichlet characters. Define a function $\xi\left(s_{1}, \ldots, s_{k}\right)$ for $\Re\left(s_{i}\right)>1(i=1, \ldots, k)$ by a convergent sum:

$$
\sum_{0<n_{1}<\ldots<n_{k}} \frac{\chi_{1}\left(n_{1}\right) \ldots \chi_{k}\left(n_{k}\right)}{\left(n_{1}+\alpha_{1}\right)^{s_{1}} \ldots\left(n_{k}+\alpha_{k}\right)^{s_{k}}} .
$$

Then $\xi$ continues meromorphically to $\mathbb{C}^{k}$. In fact, using the binomial series expansion of $(n+\beta)^{-s}=n^{-s}(1+\beta / n)^{-s}$ for each variable, we see that $\xi$ can be expressed in terms of absolutely convergent sums of multiple zeta functions. See [1] for a further study of this kind of function.

\section{References}

[1] S. Akiyama and H. Ishikawa, An analytic continuation of multiple L-functions and related zeta-functions, in: Analytic Number Theory, C. Jia and K. Matsumoto (eds.), to appear.

[2] S. Akiyama and Y. Tanigawa, Multiple zeta values at non-positive integers, submitted.

[3] T. M. Apostol, Introduction to Analytic Number Theory, Springer, 1976.

[4] T. Arakawa and M. Kaneko, Multiple zeta values, poly-Bernoulli numbers, and related zeta-functions, Nagoya Math. J. 153 (1999), 189-209.

[5] F. V. Atkinson, The mean value of the Riemann zeta-function, Acta Math. 81 (1949), 353-376.

[6] J. M. Borwein, D. M. Bradley, D. J. Broadhurst and P. Lisoněk, Combinatorial aspects of multiple zeta values, Electron. J. Combin. 5 (1998), no. 1, Research Paper $38,12 \mathrm{pp}$.

[7] D. J. Broadhurst and D. Kreimer, Association of multiple zeta values with positive knots via Feynman diagrams up to 9 loops, Phys. Lett. B 393 (1997), 403-412.

[8] H. M. Edwards, Riemann's Zeta Function, Academic Press, New York, 1974. 
[9] S. Egami, Reciprocity laws of multiple zeta functions and generalized Dedekind sums, in: Analytic Number Theory and Related Topics (Tokyo, 1991), World Sci., River Edge, NJ, 1993, 17-27.

[10] -, Introduction to multiple zeta function, lecture notes at Niigata Univ. (in Japanese); DVI and TeX files available at http://mathalg.ge.niigata-u.ac.jp/Seminar/ Intensive/Egami.html.

[11] R. C. Gunning, Introduction to Holomorphic Functions of Several Variables II, Wadsworth \& Brooks/Cole, 1990.

[12] A. Ivić, The Riemann Zeta-Function, Wiley, New York, 1985.

[13] M. Katsurada and K. Matsumoto, Asymptotic expansions of the mean values of Dirichlet L-functions, Math. Z. 208 (1991), 23-39.

[14] T. Q. T. Le and J. Murakami, Kontsevich's integral for the Homfly polynomial and relations between values of multiple zeta functions, Topology Appl. 62 (1995), 193-206.

[15] Y. Motohashi, A note on the mean value of the zeta and L-functions. I, Proc. Japan Acad. Ser. A Math. Sci. 61 (1985), 222-224.

[16] Y. Ohno, A generalization of the duality and sum formulas on the multiple zeta values, J. Number Theory 74 (1999), 39-43.

[17] E. C. Titchmarsh, The Theory of the Riemann Zeta-Function, 2nd ed. (revised by D. R. Heath-Brown), Clarendon Press, Oxford, 1986.

[18] D. Zagier, Values of zeta-functions and their applications, in: First European Congress of Mathematics, Vol. II, Birkhäuser, 1994, 497-512.

[19] —, Periods of modular forms, traces of Hecke operators, and multiple zeta values, in: Research into Automorphic Forms and L Functions (Kyoto, 1992), Sūrikaisekikenkyūsho Kōkyūroku 843 (1993), 162-170 (in Japanese).

[20] J. Q. Zhao, Analytic continuation of multiple zeta function, Proc. Amer. Math. Soc. 128 (2000), 1275-1283.

Department of Mathematics

Faculty of Science

Niigata University

Ikarashi 2-8050, Niigata 950-2181, Japan

E-mail: akiyama@mathalg.ge.niigata-u.ac.jp

Graduate School of Mathematics

Nagoya University

Chikusa-ku, Nagoya 464-8602, Japan

E-mail: tanigawa@math.nagoya-u.ac.jp
Department of Mechanical and Intelligent Systems Engineering

Faculty of Engineering

Toyama University

Gofuku 3190, Toyama 930-8555, Japan

E-mail: megami@eng.toyama-u.ac.jp

Received on 5.3.1999

and in revised form on 14.4.2000 